\title{
Management of Postpancreatectomy Hemorrhage Following Pancreaticoduodenectomy at a Tertiary Care Center in Nepal
}

\author{
Nirajan Subedi, Bishnu Kandel, Bikal Ghimire, Prasan BS Kansakar, Ramesh S Bhandari, Paleswan \\ Joshi Lakhey \\ Department of GI and General Surgery, Maharajgunj Medical Campus, Tribhuvan University Teaching Hospital, \\ Institute of Medicine, Maharajgunj, Kathmandu, Nepal
}

\section{Corresponding author:}

Paleswan Joshi Lakhey, MS, MCh

Department of GI and General Surgery, Maharajgunj Medical Campus, Tribhuvan University Teaching Hospital, Institute of Medicine, Maharajgunj, Kathmandu, Nepal

Email:lakheypj@gmail.com

Submitted : Oct 18, 2020

Accepted : Dec 8, 2020

\begin{abstract}
Introduction

Postpancreatectomy hemorrhage (PPH) is an important complication which significantly increases morbidity and mortality following pancreaticoduodenectomy (PD). This study aims to find the incidence, classification, management, and mortality associated with PPH following PD. This study also describes the changes in management and outcomes between the period of 2004-2014 and 2015-2019 in our institute as in the later time period there was use of CT angiography and interventional radiology (IR) to identify and control the bleeding site.
\end{abstract}

\section{Methods}

This is a retrospective study in which medical records of patients having PPH following PD between 20042019 were analyzed. The grading and classification were done according to the International Study Group of Pancreatic Surgery (ISGPS). Management and outcomes were analyzed using standard descriptive statistics.

\section{Results}

A total of 43 patients developed PPH out of 336 PDs. Out of 43 patients, 4 (9.3\%) had Grade A, 16 (37.2\%) had Grade B and 23 (53.5\%) had Grade C PPH. Fifteen (35\%) patients were managed conservatively, seven (16.2\%) with IR procedure and 21 (48.8\%) were reexplored. There were $16(37.2 \%)$ mortalities, out of different factors- intraoperative blood loss $>500 \mathrm{ml}$ showed the predictability for mortality $(p=0.01)$. On comparing two time periods it was seen that the PPH rate was almost similar but the mortality decreased from $58.8 \%$ to $23.07 \%$.

\section{Conclusion}

PPH following PD is associated with high mortality. Increased use of IR procedure and CT angiography can decrease the relaparotomy rates and eventually decrease mortality.

\section{Keywords}

Interventional radiology, pancreaticoduodenectomy, postpancreatectomy hemorrhage 


\section{INTRODUCTION}

$\mathrm{P}$ ancreaticoduodenectomy (PD) is a major and morbid procedure and it has been increasing in number every year with advances in imaging and increase in several centers performing it. Though the mortality has now decreased up to $5 \%$, morbidity remains up to $40 \% .^{1}$ Postoperative pancreatic fistula $(P O P F)^{2}$ remains the most common complication and also an important determinant for other complications ${ }^{3}$ like post pancreatectomy hemorrhage $(\mathrm{PPH})^{4}$, delayed gastric emptying $(D G E)^{5}$ and chyle leak ${ }^{6}$. These complications have been defined and revised by the International Study Group of Pancreatic Surgery (ISGPS). PPH though not frequently seen as POPF but significantly increases morbidity and mortality of the patient.7 Early PPH ( $<24 \mathrm{hr}$ ) is considered to be technical failure whereas delayed ( $>24 \mathrm{hr}$ ) is usually related to vessel erosion or pseudoaneurysm. The mortality/morbidity related to PPH has decreased due to the availability of CT angiography, advances in Intervention Radiology (IR) procedure like stenting and angioembolization. These procedure have led to decreased relaparotomy and mortality as well. ${ }^{8}$

In this study, we report our experience in the management of PPH at our institution. This study aimed to investigate the incidence, classification, management, and mortality associated with $\mathrm{PPH}$ after PD at a tertiary care center in Nepal. There has been change in the management and outcome of PPH between 2004 to 2014 and 2015 till 2019 in our institute and we have tried to show in our study as in the later time period there was easy availability and liberal use of $\mathrm{CT}$ angiography and interventional radiology (IR) to find and control the bleeding site.

\section{METHODS}

The study was approved by the Institutional Review Committee, Institute of Medicine (IOM), Kathmandu, Nepal. This is a retrospective study of all the patients who developed PPH following PD between 2004 to 2019 at Tribhuvan University Teaching Hospital, Maharajgunj, Kathmandu, Nepal. All the procedures were carried out at the Department of Gl and General surgery TUTH, Kathmandu. Details of patients were extracted from the hospital clinical records. All the patients with benign or malignant periampullary disease who underwent PD were included.

Any episode of bleeding, like blood in nasogastric (NG) tube, blood in the drain or passage of melenic stool was considered as PPH. Severity, timing and grade all were defined according to ISGPS. ${ }^{4}$ The classification is shown in Table 1.

All the cases of mild PPH were managed conservatively. The management of severe PPH is depicted in Figure 1. Due to the availability of the
CT angiography and angioembolization techniques, there was difference in management of severe PPH between 2004-2014 and 2015-2019. As shown in Figure 1, before easy availability of CT angiography and IR, laparotomy was only method to identify the bleeding spot and control it. In later period any severe PPH cases were stabilized and urgent CT angiography was done, if the bleeder was identified then angioembolization was done. If no active bleed was seen in CT angiography but patient had features of ongoing bleed then a conventional angiography was done. On conventional angiography if bleeding was not seen then patient was taken for laparotomy. Hence the difference in the management of PPH and outcome between these two different time period has been shown here.

Statistical analysis was performed with SPSS software (SPSS Inc., version 16 for Macintosh, IBM, Chicago, IL, USA). Continuous variables were expressed as the mean and SD or as the median with a range when appropriate. Student's t-test was used to compare the means between groups. Nonparametric tests were used when appropriate. A Chi-squared test was used for nominal data. Univariate logistic regression analysis was done for different factors like age, sex, pre-operative biliary drainage, Pancreatic consistency, diameter of main pancreatic duct, presence of POPF, time and severity of PPH, blood loss, duration of surgery and final histopathology to predict mortality in PPH

Table 1. Classification of PPH according to ISGPS

\begin{tabular}{ll}
\hline \multicolumn{1}{c}{ Class } & \multicolumn{1}{c}{ Description } \\
\hline Time of onset & \\
Early & $<24 \mathrm{hr}$ from surgery \\
Late & $>24 \mathrm{hr}$ from Surgery \\
Location & \\
Extraluminal & Bleeding inside abdominal cavity \\
Intraluminal & Intra enteric \\
Severity & \\
Mild & $\bullet$ Hb drop $<3$ g/dL \\
& $\bullet$ Mild clinical impairment \\
& $\bullet$ No therapeutic consequence - \\
& $\bullet 2-3$ PRBC if $<24$ hr from surgery \\
Severe & $\bullet$ Large blood loss \\
& $\bullet$ Hb drop $>3$ g/dL \\
& $\bullet$ Significant clinical impairment $>3$ \\
Grade C & PRBC \\
Grade & L invasive treatment \\
Grade B & Early mild \\
& Late mild \\
&
\end{tabular}




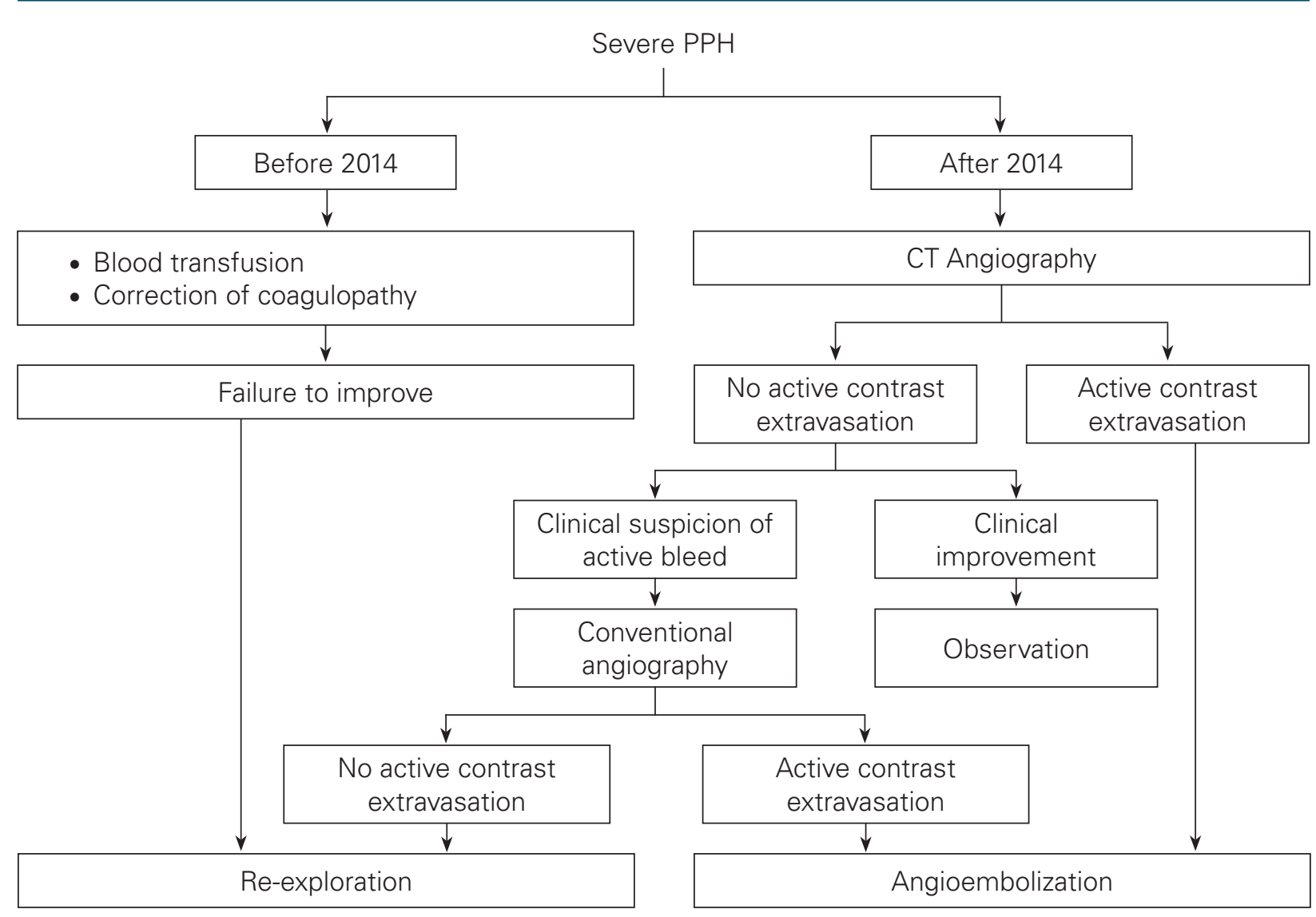

Fig 1. Management algorithm of severe $P P H$

patients. All tests were 2- tailed. P values $<0.05$ were considered statistically significant.

\section{RESULTS}

The clinical characteristics of the patients that developed PPH are summarized in Table 2. A total of 43 patients developed PPH out of 336 PDs. The mean age of those developing PPH was 51 years. Percutaneous transhepatic biliary drainage (PTBD) was preferred method of biliary drainage, done in 10(23.3\%) patients whereas Endoscopic retrograde cholangiopancreatography (ERCP) and stenting was done in two $(4.7 \%)$ cases only. No biliary drainage was done in $31(72 \%)$ patients. Mean operative duration was $412.79 \pm 294.51$ mins and mean estimated blood loss was $553.95 \pm 295.48$ $\mathrm{ml}$. The indication for PD was ampullary carcinoma in $34(79.1 \%)$ patients, distal cholangiocarcinoma in four $(11.6 \%)$ patients, carcinoma head of the pancreas (Ca HOP) in one (2.3\%) patient and other pathology were seen in four $(7 \%)$ patients.

Out of 43 patients, four patients developed Grade A PPH (9.3\%). Sixteen patients developed Grade B PPH (37.2\%) and 23 patients (53.5\%) developed Grade C PPH. POPF was associated in 35 (83.1\%) patients that had $\mathrm{PPH}$. The grade and timing of $\mathrm{PPH}$ are shown in Table 3. Location wise $19(44.2 \%)$ patients had intraluminal bleed, 22 (51\%) had extraluminal bleed and two (4.7\%) patients had both intra and extra luminal bleed.

There were total four Grade A PPH and all of them were managed conservatively. There were

Table 2. Characteristics of patients with $P P H(n=43)$

\begin{tabular}{lc}
\hline \multicolumn{1}{c}{ Characteristics } & Number \\
\hline Age (years) & $51.65 \pm 13.07$ \\
Sex $(\mathrm{F} / \mathrm{M})$ & $24 / 19$ \\
$\mathrm{BMI}\left(\mathrm{kg} / \mathrm{m}^{2}\right)$ & $21.79 \pm 3.3$ \\
Pre-operative biliary drainage & \\
No & $31(72.0 \%)$ \\
PTBD & $10(23.3 \%)$ \\
ERCP+ Stenting & $2(4.7 \%)$ \\
Pancreatic consistency & \\
Soft & $37(86) \%$ \\
Firm & $6(14 \%)$ \\
Main pancreatic duct diameter(mm) & $3.42 \pm 0.85$ \\
Duration of Surgery (mins) & $412.79 \pm 294.51$ \\
Blood loss (ml) & $553.95 \pm 295.48$ \\
Final diagnosis & \\
Ampullary carcinoma & $34(79.1 \%)$ \\
Distal cholangiocarcinoma & $4(11.6 \%)$ \\
Ca HOP & $1(2.3 \%)$ \\
Others & $4(7 \%)$ \\
\hline
\end{tabular}


Table 3. Characteristics of patients with $\mathrm{PPH}$ according to ISGPS $(n=43)$

\begin{tabular}{lc}
\hline \multicolumn{1}{c}{ Chracteristics } & Frequency \\
\hline PPH Time & $12(27.9 \%)$ \\
Early & $31(72.1 \%)$ \\
Late & \\
PPH Severity & $13(30.2 \%)$ \\
Mild & $30(69.8 \%)$ \\
Severe & \\
Location & $19(44.2 \%)$ \\
Intraluminal & $22(51.1 \%)$ \\
Extraluminal & $2(4.7 \%)$ \\
Both & \\
PPH Grade & $4(9.3 \%)$ \\
Grade A & $16(37.2 \%)$ \\
Grade B & $23(53.5 \%)$ \\
Grade C & \\
Associated with POPF & \\
Yes & $85(83.1 \%)$ \\
No & $8(16.9 \%)$ \\
\hline
\end{tabular}

a total of 16 Grade B PPH Patients. Out of those 16 Patients, seven patients had early severe PPH and nine patients had late mild PPH. Six (85.7\%) out of seven early severe PPH patients were reexplored, one $(14.3 \%)$ patient was managed with glue embolization. There were two (28.5\%) mortalities among six reexplored patients. All of the late mild $\mathrm{PPH}$ cases were managed conservatively. There were three (33.3\%) mortalities in the late mild group. There were a total of $23 \mathrm{Grade} \mathrm{C} \mathrm{PPH}$ patients. Two (8.6\%) were managed conservatively, six (26.0\%) patients were managed with IR procedures and $15(65.4 \%)$ were reexplored. There were a total of $11(47.8 \%)$ mortalities in Grade C PPH group, nine $(81.3 \%)$ of the 15 reexplored patient had mortality. One $(16.67 \%)$ of the six patients that was managed with an IR procedure had mortality. One $(50 \%)$ of the two patients that was managed conservatively had mortality.

There were total 16 (37.2\%) mortalities of the patients having $\mathrm{PPH}$. Amongst various factors as shown in Table 4 that were considered for predicting mortality in $\mathrm{PPH}$ patients, blood loss more than $500 \mathrm{ml}$ showed the predictability of mortality after undergoing surgery. $(p=0.01 O R=5.8(C l-1.5-22.7)$ This signifies that the chances of mortality were 5.8 times higher in patients having intraoperative blood loss more than $500 \mathrm{ml}$ in comparison to surgeries with blood loss less than $500 \mathrm{ml}$. However, other risk factors did not show any significant association.

We have compared our data between 20042014 and 2015-2019 (Table 5). The number of PDs has almost doubled in the last five years, though the PPH rate is almost similar $13.2 \%$ and $12.5 \%$ respectively. In the last five years, use of IR
Table 4. Predictive risk factors of mortality in patients with $P P H(n=16)$

\begin{tabular}{|c|c|c|}
\hline Risk factors & No. & $p$-value/OR \\
\hline \multicolumn{3}{|l|}{ Age (years) } \\
\hline$<50$ & 8 & $p=0.28$ \\
\hline$\geq 50$ & 8 & $\mathrm{OR}=0.5(0.14-1.7)$ \\
\hline \multicolumn{3}{|l|}{ Sex } \\
\hline Female & 7 & $p=0.22$ \\
\hline Male & 9 & $\mathrm{OR}=2.18(0.62-7.7)$ \\
\hline \multicolumn{3}{|c|}{ Ampullary Malignancy } \\
\hline No & 4 & $p=0.61$ \\
\hline Yes & 12 & $\mathrm{OR}=0.68(0.15-3.02)$ \\
\hline \multicolumn{3}{|c|}{ Pancreatic Consistency } \\
\hline Soft & 13 & $p=0.48$ \\
\hline Firm & 3 & $O R=1.84(0.32-10.4)$ \\
\hline \multicolumn{3}{|l|}{ MPD>3 mm } \\
\hline No & 11 & $p=0.28$ \\
\hline Yes & 5 & $\mathrm{OR}=0.49(0.13-1.8)$ \\
\hline \multicolumn{3}{|l|}{ POPF } \\
\hline No & 3 & $p=0.78$ \\
\hline Yes & 13 & $\mathrm{OR}=1.23(0.26-5.8)$ \\
\hline \multicolumn{3}{|l|}{ PPHTime } \\
\hline Early & 2 & $p=0.9$ \\
\hline Late & 14 & $\mathrm{OR}=4.1(0.77-21.9 .6)$ \\
\hline \multicolumn{3}{|l|}{ PPH severity } \\
\hline Mild & 3 & $p=0.21$ \\
\hline Severe & 13 & $\mathrm{OR}=2.5(0.58-11.1)$ \\
\hline \multicolumn{3}{|l|}{ Blood loss $>500 \mathrm{ml}$} \\
\hline No & 6 & $p=0.01$ \\
\hline Yes & 10 & $\mathrm{OR}=5.8(1.5-22.7)$ \\
\hline \multicolumn{3}{|l|}{ Preop Drainage } \\
\hline No & 12 & $p=0.74$ \\
\hline Yes & 4 & $\mathrm{OR}=0.79(0.19-3.2)$ \\
\hline \multicolumn{3}{|c|}{ Surgery time $>300$ mins } \\
\hline No & 5 & $p=0.88$ \\
\hline Yes & 11 & $\mathrm{OR}=1.1(0.29-4.1)$ \\
\hline
\end{tabular}

Table 5. Incidence, grade and management of PPH between 2004-2014 and 2015-2019

\begin{tabular}{lcc}
\hline \multicolumn{1}{c}{ Details } & 2004-2014 & 2015-2019 \\
\hline Total PD & 128 & 208 \\
PPH & $17(13.2 \%)$ & $26(12.5 \%)$ \\
Grade of PPH & & \\
Grade A & - & 4 \\
Grade B & 7 & 9 \\
Grade C & 10 & 13 \\
Management & & \\
Conservative & $7(41.2 \%)$ & $8(30.7 \%)$ \\
Angioembolisation & - & $7(26.9 \%)$ \\
Reexploration & $10(58.8 \%)$ & $11(42.3 \%)$ \\
Mortality & $10(58.8 \%)$ & $6(23.07 \%)$ \\
\hline
\end{tabular}


procedures have decreased the relaparotomy rate of $58.8 \%$ vs $42.3 \%$. Out of seven patients that had IR intervention, five(71.4\%) had coil embolization done, glue embolization and gel foam embolization was done in one(14.3\%) patient each. Though PPH rates are similar, mortality rate has decreased from $58.8 \%$ to $23.07 \%$.

\section{DISCUSSION}

Pancreaticoduodenectomy is one of the major surgeries done in surgical gastroenterology units. TUTH is one of the high volumes centers in Nepal and on average more than 50 PDs are performed every year. One of the unique features is that we have more ampullary cases than carcinoma head of the pancreas, so the pancreas we operate are soft and have non dilated pancreatic duct and also dunking method of pancreaticojejunostomy (PJ) is done in most of the cases.

$\mathrm{PPH}$ rate in our center is $12.7 \%$ which is higher than that shown by Alvaro et $\mathrm{al}^{7}$, Gallego et . $\mathrm{al}^{8}$ and Feng $^{9}$ et al which is 3\%,6.8\% and $8.7 \%$ respectively. There can be multiple explanations for high $\mathrm{PPH}$, first the pancreas we operate have soft consistency leading to higher chances of POPF and eventually $\mathrm{PPH}$. Second is the dunking method of PJ, as shown in RECOPANC trial where pancreatogastrostomy (PG) was compared with $P J$ and $P G$ in this study was done by dunking method, the PG group had higher incidence of intraluminal $\mathrm{PPH} .{ }^{10}$

In our series, we had Grade C PPH as the most frequent type. Incidence of Grade B/C PPH was $90.7 \%$ of all the $\mathrm{PPH}$ patients. Okoda et.al ${ }^{11}$ had $\mathrm{B}$ and $\mathrm{CPPH}$ rate of $38 \%$ whereas in series by Ansari et $\mathrm{al}^{12}$ it was $6.8 \%$ of overall patients. Most of the studies consider Grade B/C as clinically relevant $\mathrm{PPH}$ and consider Grade A PPH just as part of a normal postoperative course. Hence there is a suggestion to reconsider the grading of $\mathrm{PPH}$ like that of POPF.

The mortality rate in our series was $37.2 \%$ of those who had PPH. The mortality rate is higher than Asari et $\mathrm{al}^{13}$, Izumo et al ${ }^{14}$ with $20 \%$ and $11 \%$ respectively but the mortality rate was almost similar to Wolk et.al ${ }^{15}$ of $32 \%$. Amongst the factors that predicts mortality in patients that had $\mathrm{PPH}$, we found intra operative blood loss $>500 \mathrm{ml}$ to be an independent predictor. Wellener et $\mathrm{al}^{16}$ in his series showed that Age $>79, \mathrm{BMI}>26$, Bilirubin $>1.25$ and presence of POPF to be an independent predictor of mortality.

In our cohort of patients, ampullary carcinoma was the most common final diagnosis which was in $79.7 \%$ patients and carcinoma head of the pancreas was only in $2.3 \%$ patients. In a study by Ansari et.al ${ }^{12}$ the most common histopathology was PDAC 33.8\% and ampullary carcinoma was in $9 \%$ patients. Similarly, Wellner et.al ${ }^{16}$ also showed that pancreatic ductal adenocarcinoma (PDAC) was the most common type in $33.8 \%$ cases and periampullary was in $14.6 \%$ cases but in the study by Wolk et.al ${ }^{15}$, ampullary carcinoma was seen as the most common type, seen in $74.8 \%$ cases and PDAC was seen only in $18.3 \%$ as final diagnosis. Most of the data from the west shows PDAC as the most common type of histopathology whereas we have ampullary carcinoma as the most common pathology. Hence our pancreas are softer so, have higher chances of POPF and its sequalae like PPH.

In our series $81.3 \%$ cases that had PPH had POPF and $80.6 \%$ of the late PPH patients had POPF. Also in the study by Ansari et al and Wellner et al POPF was significantly associated with PPH. POPF has been closely related to $\mathrm{PPH}$, the amylase rich fluid erodes the branch of blood vessels, causes pseudoaneurysm or intra-abdominal collection which can cause pressure effect eventually leading to haemorrhage. ${ }^{4}$

In a study the authors argued that Grade B PPH is a heterogeneous group. In Grade B both early severe and delayed Mild PPH are included. They argued that management and the clinical course of two groups is entirely different hence advocating for a reappraisal of classification. ${ }^{8}$ In our Study also, out of 16 patients that had Grade B PPH, seven out of eight patients in the early severe group were reexplored whereas rest nine that had late mild were managed conservatively.

In our center, there has been a shift in paradigm in the management of PPH. The factors may be more experienced, more vigilant working staff and good support of IR. The mortality due to PPH has decreased from $58.8 \%$ to $23 \%$. In a study done by Wolk, ${ }^{15}$ the use of angiography has been significantly increased between period 1994 to 2009 and 2010 to 2014 but the relaparotomy and mortality rate were similar $45.6 \%$ vs $42.1 \%$ and $26.2 \%$ and $28.2 \%$.

Being a retrospective study our study carries all the inherent problems like loss of data, selection bias and loss to follow up, still it gives some insight about a lethal complication of a complex surgery in a developing country. We are still in the stage of evolution, our preferred method for biliary drainage is PTBD rather than ERCP. Five years back reexploration was the only method of addressing severe PPH. We are still far away from minimally invasive pancreatic surgery. With an increased number of cases, knowledge and good support from Department of Critical Care and Department of Intervention Radiology, we have decreased the mortality rates but morbidity remains high.

\section{CONCLUSION}

Postpancreatectomy hemorrhage following pancreaticoduodenectomy is associated with high 
mortality. Increased use of IR procedure and CT angiography can decrease the relaparotomy rates and eventually decrease mortality.

\section{CONFLICT OF INTEREST}

None declared.

\section{REFERENCES}

1. He J, Ahuja N, Makary MA, et al. 2564 resected periampullary adenocarcinomas at a single institution: trends over three decades. HPB. 2014 Jan;16(1):83-90.

2. Bassi C, Marchegiani G, Dervenis C, et al. The 2016 update of the International Study Group (ISGPS) definition and grading of postoperative pancreatic fistula: 11 Years After. Surgery. 2017 Mar;161(3):584-91.

3. Vollmer CM. The economics of pancreas surgery. Surg Clin North Am. 2013 Jun;93(3):711-28.

4. Wente MN, Veit JA, Bassi C, et al. Postpancreatectomy hemorrhage (PPH)-An International Study Group of Pancreatic Surgery (ISGPS) definition. Surgery. 2007 Jul; 142(1):20-5.

5. Wente MN, Bassi C, Dervenis C, et al. Delayed gastric emptying (DGE) after pancreatic surgery: A suggested definition by the International Study Group of Pancreatic Surgery (ISGPS). Surgery. 2007 Nov; 142(5):761-8.

6. Besselink $M G$, van Rijssen $L B$, Bassi $C$, et al. Definition and classification of chyle leak after pancreatic operation: A consensus statement by the International Study Group on Pancreatic Surgery. Surgery. 2017 Feb;161(2):365-72.

7. Correa-Gallego C, Brennan MF, D'Angelica MI, et al. Contemporary Experience with Postpancreatectomy Hemorrhage: Results of 1,122 Patients Resected between 2006 and 2011. J Am Coll Surg. 2012
Nov:215(5):616-21.

8. Duarte Garcés AA, Andrianello S, Marchegiani G, et al. Reappraisal of post-pancreatectomy hemorrhage (PPH) classifications: do we need to redefine grades A and B? HPB. 2018 Aug;20(8):702-7.

9. Feng J, Chen Y-L, Dong J-H, et al. Post-pancreaticoduodenectomy hemorrhage: risk factors, managements and outcomes. Hepatobiliary Pancreat Dis Int HBPD INT. 2014 Oct;13(5):513-22.

10. Keck $T$, Wellner UF, Bahra $M$, et al. Pancreatogastrostomy Versus Pancreatojejunostomy for RECOnstruction After PANCreatoduodenectomy (RECOPANC, DRKS 00000767): Perioperative and Long-term Results of a Multicenter Randomized Controlled Trial. Ann Surg. 2016 Mar;263(3):440-9.

11. Okada K, Murakami Y, Uemura K, et al. Flooring the Major Vessels with Falciform Ligament to Prevent Post-Pancreatectomy Hemorrhage. World J Surg. 2020 0ct;44(10):3478-85.

12. Ansari $D$, Tingstedt $B$, Lindell $G$, et al. Hemorrhage after Major Pancreatic Resection: Incidence, Risk Factors, Management, and Outcome. Scand I Surg SJS Off Organ Finn Surg Soc Scand Surg Soc. 2017 Mar;106(1):47-53.

13. Asari S, Matsumoto I, Toyama $H$, et al. Recommendation of treatment strategy for postpancreatectomy hemorrhage: Lessons from a single-center experience in 35 patients. Pancreatology. 2016 May;16(3):454-63.

14. Izumo W, Higuchi R, Yazawa T, et al. Evaluation of preoperative risk factors for postpancreatectomy hemorrhage. Langenbecks Arch Surg. 2019 Dec;404(8):967-74.

15. Wolk S, Grützmann R, Rahbari NN, et al. Management of clinically relevant postpancreatectomy hemorrhage (PPH) over two decades - A comparative study of 1450 consecutive patients undergoing pancreatic resection. Pancreatology. 2017 Nov; 17(6):943-50.

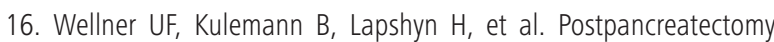
Hemorrhage-Incidence, Treatment, and Risk Factors in Over 1,000 Pancreatic Resections. J Gastrointest Surg. 2014 Mar;18(3):464-75. 